\title{
Regularity of Weakly Subelliptic F-Harmonic Maps
}

\author{
Zhen-Rong Zhou ${ }^{1}$ \\ ${ }^{1}$ Department of Mathematics, Central China Normal University, Wuhan, P. R. China \\ Correspondence: Zhen-Rong Zhou, Department of Mathematics, Central China Normal University, Wuhan 430079, \\ P. R. China. E-mail: zrzhou@mail.ccnu.edu.cn
}

Received: June 16, 2013 Accepted: July 2, 2013 Online Published: July 23, 2013

doi:10.5539/jmr.v5n3p26 URL: http://dx.doi.org/10.5539/jmr.v5n3p26

Research supported by National Science Fundation of China No.10871149

\begin{abstract}
Let $\Omega \subseteq \mathbb{R}^{m}$ be a bounded domain, $X=\left\{X_{1}, X_{2}, \cdots, X_{k_{0}}\right\}$ be a Hömander family of vector fields on $\Omega$ whose homogeneous dimension is $Q$. In this paper, we improve the dual inequality obtained by P. Hajlasz and P. Strzelecki in 1998, and take use of it to discuss regularity of weakly subelliptic F-harmonic maps into Riemannian manifolds with transitive isometric transformation groups.
\end{abstract}

Keywords: subelliptic F-harmonic map, regularity

Classification of MR2000: 58E20

\section{Introduction, Main Results}

Let $\Omega$ be a bounded domain of $\mathbb{R}^{m}, X=\left\{X_{1}, X_{2}, \cdots, X_{k_{0}}\right\}$ a family of $C^{\infty}$ vector fields on $\Omega$. If the bracket products of $\left\{X_{\alpha}, \alpha=1, \cdots, k_{0}\right\}$ span the tangent space of $\Omega$ at every point, we call $X$ to satisfy the Hormander's condition, or to be a Hormander family of vector fields. If $\gamma:[0, a] \rightarrow \Omega$ is a piecewise smooth curve, and $\gamma^{\prime}(t)=\sum_{\alpha=1}^{k_{0}} c^{\alpha}(t) X_{\alpha}(\gamma(t))$ for almost all $t \in[0, a]$, then $\gamma$ is called horizontal (with respect to $X$ ). The set of all horizontal curves in $\Omega$ is denoted by $\mathcal{H}$. Define a metric on $\Omega$ as follows:

$$
d_{c c}(x, y)=\inf _{\gamma \in \mathcal{H}}\{T \mid \gamma(0)=x, \gamma(T)=y\} .
$$

By the Chow's theorem, the Hormander's condition guarantees that any two points can be connected by horizontal curves. Therefore, for any $x, y \in \Omega$, we have $d_{c c}(x, y)<\infty$, i.e. $\left(\Omega, d_{c c}\right)$ is a metric space. We call the metric $d_{c c}$ the Carnot-Caratheodory metric, or the CC-metric for short. A CC-metric ball with center $x$ and radius $r$ is denoted by $B_{r}(x)$, and its Lebesgue measure by $\left|B_{r}(x)\right|$. Then there holds the following doubling inequality:

$$
\left|B_{2 r}(x)\right| \leq C_{d}\left|B_{r}(x)\right|
$$

for small $r$, where $C_{d}$ is a constant called a doubling constant.

Let $\mathbb{R}^{K}$ be a $K$ dimensional Euclidean space, and define a Sobolev space as

$$
M^{1, p}\left(\Omega, \mathbb{R}^{K}\right) \equiv\left\{u: \Omega \rightarrow \mathbb{R}^{K} \mid u \in L^{p} ; X_{\alpha} u \in L^{p}, \alpha=1, \cdots k_{0}\right\} .
$$

Let $M_{0}^{1, p}\left(\Omega, \mathbb{R}^{K}\right)$ stand for the closure of $C_{0}^{\infty}\left(\Omega, \mathbb{R}^{K}\right)$ in $M^{1, p}\left(\Omega, \mathbb{R}^{K}\right)$ with respect to the following norm:

$$
\|u\|_{M^{1, p}} \equiv\left(\int_{\Omega}|u|^{p}+\int_{\Omega}|X u|^{p}\right)^{1 / p}
$$

where $|X u|=\sqrt{\sum\left|X_{\alpha} u\right|^{2}}$. In this paper, we adopt the convention of summation. The range of indexes $\alpha, \beta$ is $\left\{1, \cdots, k_{0}\right\}$.

Let $N$ be a compact Riemannian manifold. By Nash's imbedding theorem, we can assume that $N$ is a submanifold of the Euclidean space $\mathbb{R}^{K}$ (for some positive integer $K$ ) without loss of generality. Define subelliptic $F$-energy functional of $u$ as 


$$
\mathcal{E}_{F}(u) \equiv \int_{\Omega} F\left(\frac{|X u|^{2}}{2}\right)
$$

for a smooth map $u: M \rightarrow N$, where $F:[0, \infty) \rightarrow[0, \infty)$ is a smooth function. The critical points of $\mathcal{E}_{F}(\cdot)$ are called subelliptic $F$-harmonic maps (with respect to $X$ ). If $F(t)=t, \frac{1}{p}(2 t)^{p}$, $\exp (2 t)$, then the subelliptic $F$-harmonic maps are called subelliptic harmonic maps, subelliptic $p$-harmonic maps, subelliptic exponential harmonic maps, respectively. Especially, when $X_{i}=\frac{\partial}{\partial x_{i}}$, subelliptic $F$-harmonic maps are the originary $F$-harmonic maps. Therefore, subelliptic $F$-harmonic maps are the generalization of $F$-harmonic maps which cover harmonic maps, $p$-harmonic maps, exponential harmonic maps, etc.

Let $M^{1, p}(\Omega, N)=\left\{u \in M^{1, p}\left(\Omega, \mathbb{R}^{K}\right): u(x) \in N\right.$ a.e. in $\left.\Omega\right\}$. If $F(t) \leq C t^{\frac{p}{2}}$, and $u \in M^{1, p}(\Omega, N)$ is a critical point of the $F$-energy functional $\mathcal{E}_{F}(u)$, then $u$ is called a weakly subelliptic $F$-harmonic map.

Subelliptic harmonic maps are introduced by Jost and Xu in 1998. It is known that a weakly harmonic map from a surface is regular. This result is proven by Helein (see Helein, 1990, 1991a, 1991b). Strzelecki (1994) generalizes partially the Helein's result, and proves that weakly $p$-harmonic maps from $p$ dimensional domains into spheres are regular. All these regularities are obtained by taking use of the Hardy space theory. A natural question is: Are the above conclusions true for subelliptic harmonic maps? In this case, the Hardy space theory are not valid more. However, Hajłasz and Strzelecki in 1998 establish a dual inequality, which is called H-S dual inequality here, and use it to get a regularity of weakly subelliptic $Q$-harmonic maps into spheres, where $Q$ is the homogeneous dimension of the domains. E. Barletta and S. Dragomir also take use of H-S dual inequality to prove that weakly subelliptic $F$-harmonic maps into spheres are regular, if $F^{\prime}$ has $\left(\frac{Q-2}{2}\right)$-power growth (see Barletta \& Dragomir, 2004), and hence generalize the Hajłasz-Strzelecki's regularity. In this paper, we improve the dual inequality of Hajłasz-Strzelecki (1998), and apply it to obtain a regularity lemma of weak solutions of a subelliptic PDE of divergent type. As an application, we get a regularity of weakly subelliptic $F$-harmonic maps into Riemannian manifolds with transitive isometric transformation groups.

The main theorems of this paper are stated here.

Lemma 1 Let $A_{\alpha \beta}=A_{\alpha \beta}(x, u, X u) \sim|X u|^{Q-2} \delta_{\alpha \beta}$ and $\xi=\xi(x, u, X u)$ satisfy $X^{*} \xi=0$ and $|\xi| \leq|X u|^{Q-1}$, and $Y^{i}$ be some functions, then any weak solution $u \in M^{1, Q}\left(\Omega, \mathbb{R}^{K}\right)$ of the following subelliptic PDE system

$$
\sum X_{\alpha}^{*}\left(A_{\alpha \beta} X_{\beta} u^{i}\right)=X^{*}\left(\xi Y^{i}(u)\right)
$$

is Holder continuous, where, $X^{*} \xi$ is the subelliptic divergence of $\xi$ and $Q$ is the homogeneous dimension of $\Omega$.

Take $A_{\alpha \beta}(x, u, X u)=F^{\prime}\left(\frac{|X u|^{2}}{2}\right) \delta_{\alpha \beta}$. Then the condition $A_{\alpha \beta}(x, u, X u) \sim|X u|^{Q-2} \delta_{\alpha \beta}$ becomes as $F^{\prime}(t) \sim t^{\frac{Q-2}{2}}$. Therefore we have

Theorem 2 Assume that $F^{\prime}(t) \sim t^{\frac{Q-2}{2}}$ and $N$ is a compact Riemannian manifold on which the isometric transformation group acts transitively, then, any weakly subelliptic $F$-harmonic map $u \in M^{1, Q}(\Omega, N)$ is Holder continuous.

This theorem is a generalization of theorems in Barletta and Dragomir (2004) and Hajlasz and Strzelecki (1998).

\section{Doubling Spaces}

Let $(\Omega, \rho, \mu)$ be a metric-measure space, $B(x, r)$ a ball of $\Omega$ with center at $x$ and radius $r$ and $n B(x, r)=B(x, n r)$. If there exists a constant $C_{d}$ such that

$$
\mu(B(x, 2 r)) \leq C_{d} \mu(B(x, r)),
$$

then we call $(\Omega, \rho, \mu)$ a doubling space, and $C_{d}$ a doubling constant.

If $\Omega$ is an open subset of the Euclidean space, $d_{c c}$ the CC-metric on $\Omega$ associated with a Hormander family of vector fields and $\mu$ the Lebesgue measure, then $\left(\Omega, d_{c c}, \mu\right)$ is a doubling space.

Lemma 3 If $(\Omega, \rho, \mu)$ is a doubling space, and $r \leq r_{0}$, then we have

$$
\frac{\mu(B(x, r))}{\mu\left(B\left(x, r_{0}\right)\right)} \geq C_{d}^{-1}\left(\frac{r}{r_{0}}\right)^{Q},
$$

where $Q=\log _{2} C_{d}$.

Proof. There exists a positive integer $n$, such that $\frac{1}{2^{n}} \leq \frac{r}{r_{0}} \leq \frac{1}{2^{n-1}}$, i.e. $2^{n-1} r \leq r_{0} \leq 2^{n} r$. According to the doubling inequality, we have

$$
\mu\left(B\left(x, r_{0}\right)\right) \leq \mu\left(B\left(x, 2^{n} r\right)\right) \leq\left(C_{d}\right)^{n} \mu(B(x, r)),
$$


from which we get $\frac{\mu(B(x, r))}{\mu\left(B\left(x, r_{0}\right)\right)} \geq \frac{1}{\left(C_{d}\right)^{n}}$. Because $2^{n-1} r \leq r_{0} \leq 2^{n} r$, we have $\log _{2} \frac{r_{0}}{r} \leq n \leq 1+\log _{2} \frac{r_{0}}{r}$. Hence we have

$$
\begin{aligned}
\left(C_{d}\right)^{-n} & \geq\left(C_{d}\right)^{-\left(1+\log _{2} \frac{r_{0}}{r}\right)} \\
& =\left(C_{d}\right)^{-1}\left(C_{d}\right)^{\log _{2} \frac{r}{r_{0}}} \\
& =\left(C_{d}\right)^{-1} 2^{\log _{2} \frac{r}{r_{0}} \log _{2} C_{d}} \\
& =\left(C_{d}\right)^{-1}\left(\frac{r}{r_{0}}\right)^{\log _{2} C_{d}} \\
& =\left(C_{d}\right)^{-1}\left(\frac{r}{r_{0}}\right)^{Q} .
\end{aligned}
$$

The lemma follows from (8) and (9).

Note that, if $\Omega$ is a bounded doubling space, taking $r_{0}=\operatorname{diam} \Omega$ in (7) yields

$$
\mu(B(x, r)) \geq \frac{1}{C_{d}} \frac{\mu(\Omega)}{(\operatorname{diam} \Omega)^{\mathrm{Q}}} r^{Q} .
$$

Let $\rho$ be a metric on $\Omega$ and $\partial \Omega \neq \emptyset$, and let

$$
r(x)=\frac{1}{1000} \rho(x, \partial \Omega)
$$

Then we have

Lemma 4 Let $\mathcal{B}=\{B(x, r(x)): x \in \Omega\}$. If $B_{1}=B\left(x_{1}, r\left(x_{1}\right)\right), B_{2}=B\left(x_{2}, r\left(x_{2}\right)\right) \in \mathcal{B}$, and $k B_{1} \cap l B_{2} \neq \emptyset$ for some $k, l$, where $k, l<1000$, then $r\left(x_{1}\right)$ and $r\left(x_{2}\right)$ are comparable, i.e. there exist constants $C_{1}, C_{2}$, depending only on $k$, $l$, such that

$$
C_{1} r\left(x_{2}\right) \leq r\left(x_{1}\right) \leq C_{2} r\left(x_{2}\right)
$$

Proof. $\forall x \in B_{1} \cap B_{2}$ and $\forall w \in \partial \Omega$, let $r_{i}=r\left(x_{i}\right), i=1,2$. Then we have

$$
\begin{aligned}
r_{1} & \leq \frac{1}{1000} \rho\left(x_{1}, w\right) \leq \frac{1}{1000}\left[\rho\left(x_{1}, x_{2}\right)+\rho\left(x_{2}, w\right)\right] \\
& \leq \frac{1}{1000}\left[k r_{1}+l r_{2}+\rho\left(x_{2}, w\right)\right] .
\end{aligned}
$$

Taking infimums for $w$, we have

$$
r_{1} \leq \frac{1}{1000}\left(k r_{1}+l r_{2}+1000 r_{2}\right)=\frac{1}{1000}\left[k r_{1}+(1000+l) r_{2}\right]
$$

from which we get $r_{1} \leq \frac{1000+l}{1000-k} r_{2}$.

Similarly, we have $r_{2} \leq \frac{1000+k}{1000-l} r_{1}$. Therefore we obtain $\frac{1000-l}{1000+k} r_{2} \leq r_{1} \leq \frac{1000+l}{1000-k} r_{2}$.

Lemma 5 There exists a sequence $\left\{x_{i} \in \Omega \mid i \in I \subseteq \mathbb{N}\right\}$, such that the members of the family of balls

$$
\mathcal{B}_{0}=\left\{B_{i}=B\left(x_{i}, r\left(x_{i}\right)\right)\right\}
$$

are pairwise disjoint, and that $3 \mathcal{B}_{0}=\left\{3 B_{i}\right\}$ covers $\Omega$.

Furthermore, if $\mu$ is doubling, then the covering multiplicity of $3 \mathcal{B}_{0}$ is not more than a positive integer $N_{3}$ which depends only on the doubling constant.

Generally, if $k \mathcal{B}_{0}$ is a covering of $\Omega$ for some $k \geq 3$, and $\mu$ doubling, then, the covering multiplicity of $k \mathcal{B}_{0}$ is not more than a positive integer $N_{k}$ which depends only on the doubling constant.

Proof. We can find a family of maximal pairwise disjoint balls $\mathcal{B}_{0}=\left\{B_{i} \mid i \in I \subseteq \mathbb{N}\right\}$ in $\mathcal{B}=\{B(x, r(x)) \mid x \in \Omega\}$, that is to say that if we add a ball of $\mathcal{B}$ to the family $\mathcal{B}_{0}$, then there must be two balls which intersect each other. It is sufficient to check that $3 \mathcal{B}_{0}=\left\{3 B_{i}\right\}$ is a covering of $\Omega$. 
Use reduction to absurdity. If it were false, there would be an $x_{0} \in \Omega$, such that $\forall i \in I$ we have $x_{0} \notin 3 B_{i}$. Now we prove that $B_{0}=B\left(x_{0}, r\left(x_{0}\right)\right)$ is disjoint with any member of $\mathcal{B}_{0}$, and hence a contradiction is produced since $\mathcal{B}_{0}$ is a family of maximal disjoint balls. In fact, $\forall y \in B_{0}$ we have

$$
\rho\left(y, x_{i}\right) \geq \rho\left(x_{0}, x_{i}\right)-\rho\left(x_{0}, y\right) \geq \rho\left(x_{0}, x_{i}\right)-r\left(x_{0}\right) .
$$

Taking a point $w$ arbitrarily at $\partial \Omega$, then we have

$$
r\left(x_{0}\right) \leq \frac{1}{1000} \rho\left(x_{0}, w\right) \leq \frac{1}{1000}\left[\rho\left(x_{0}, x_{i}\right)+\rho\left(x_{i}, w\right)\right] .
$$

Inserting (17) into (16) yields

$$
\rho\left(y, x_{i}\right) \geq \frac{999}{1000} \rho\left(x_{0}, x_{i}\right)-\frac{1}{1000} \rho\left(x_{i}, w\right) .
$$

Take infimums at the two ends for $w$. We get

$$
\rho\left(y, x_{i}\right) \geq \frac{999}{1000} \rho\left(x_{0}, x_{i}\right)-\frac{1}{1000} \rho\left(x_{i}, \partial \Omega\right)=\frac{999}{1000} \rho\left(x_{0}, x_{i}\right)-r\left(x_{i}\right) .
$$

Because we have assumed that $x_{0} \notin 3 B_{i}$ for any $i \in I$, we have $\rho\left(x_{0}, x_{i}\right) \geq 3 r\left(x_{i}\right)$ from which we have

$$
\rho\left(y, x_{i}\right) \geq \frac{999}{1000} 3 r\left(x_{i}\right)-r\left(x_{i}\right)=\frac{1997}{1000} r\left(x_{i}\right)>r\left(x_{i}\right),
$$

which implies that $y \notin B_{i}$. Hence $\forall i \in I, B_{0}$ is disjoint with $B_{i}$, which conflicts to the maximum.

Next, let us prove that the covering multiplication at each point is less than a constant $N_{3}$.

Take a point $x \in \Omega$ arbitrarily, and let $\mathcal{B}_{x}$ be a subset of $3 \mathcal{B}_{0}$ which cover $x$, i.e.

$$
\mathcal{B}_{x}=\left\{3 B_{j} \in 3 \mathcal{B}_{0} \mid x \in 3 B_{j}\right\} .
$$

In the following, we prove that the cardinal number of $\mathcal{B}_{x}$ is not more than a positive integer $N_{3}$ which is dependent only on the doubling constant. By Lemma 4 , for any $3 B_{i}=B\left(x_{i}, 3 r\left(x_{i}\right)\right)$ and $3 B_{j}=B\left(x_{j}, 3 r\left(x_{j}\right)\right) \in \mathcal{B}_{x}$, there exists a positive constant $C$, such that $C^{-1} r\left(x_{i}\right) \leq r\left(x_{j}\right) \leq \operatorname{Cr}\left(x_{i}\right)$ since $3 B_{i} \cap 3 B_{j} \neq \emptyset$. Hence for all $y \in B_{j}$, we have

$$
\begin{aligned}
\rho\left(x_{i}, y\right) & \leq \rho\left(x_{i}, x_{j}\right)+\rho\left(x_{j}, y\right) \\
& \leq 3 r\left(x_{i}\right)+3 r\left(x_{j}\right)+r\left(x_{j}\right) \\
& \leq(3+4 C) r\left(x_{i}\right),
\end{aligned}
$$

which shows that $B_{j} \subseteq(3+4 C) B_{i}$. So we get $\bigcup_{3 B_{j} \in \mathcal{B}_{x}} B_{j} \subseteq(3+4 C) B_{i}$. Since $B_{j}$ 's are disjoint, we have

$$
\sum_{3 B_{j} \in \mathcal{B}_{x}} \mu\left(B_{j}\right) \leq \mu\left((3+4 C) B_{i}\right)
$$

Taking $k=\left[\log _{2}(3+4 C)\right]+1$, we have $3+4 C \leq 2^{k}$. Hence by the doubling condition, we have

$$
\sum_{3 B_{j} \in \mathcal{B}_{x}} \mu\left(B_{j}\right) \leq \mu\left((3+4 C) B_{i}\right) \leq \mu\left(2^{k} B_{i}\right) \leq\left(C_{d}\right)^{k} \mu\left(B_{i}\right) .
$$

Summing both ends for $i$ such that $3 B_{i} \in \mathcal{B}_{x}$, we get

$$
\left|\mathcal{B}_{x}\right| \sum_{3 B_{j} \in \mathcal{B}_{x}} \mu\left(B_{j}\right) \leq\left(C_{d}\right)^{k} \sum_{3 B_{i} \in \mathcal{B}_{x}} \mu\left(B_{i}\right),
$$

from which we have $\left|\mathcal{B}_{x}\right| \leq\left(C_{d}\right)^{k} \equiv N_{3}$.

Lemma 6 Let $B_{i}=B\left(x_{i}, r_{i}\right), i=1,2$ be two balls of a doubling space $(\Omega, \rho, \mu)$. If $r_{1} \approx r_{2}$, and $B_{1} \cap B_{2} \neq \emptyset$, then we have $\mu\left(B_{1}\right) \approx \mu\left(B_{2}\right)$, i.e. $A \mu\left(B_{2}\right) \leq \mu\left(B_{1}\right) \leq B \mu\left(B_{2}\right)$ for some constants $A$ and $B$. 
Proof. Let $C^{-1} r_{2} \leq r_{1} \leq C r_{2}$. For any $y \in B_{2}$, we have

$$
\rho\left(x_{1}, y\right) \leq \rho\left(x_{1}, x_{2}\right)+\rho\left(x_{2}, y\right) \leq\left(r_{1}+r_{2}\right)+r_{2} \leq(1+2 C) r_{1},
$$

which shows that $B_{2} \subseteq(1+2 C) B_{1} \subseteq 2^{k} B_{1}$, where $k=\left[\log _{2}(1+2 C)\right]+1$. From the doubling inequality we have

$$
\mu\left(B_{2}\right) \leq \mu\left(2^{k} B_{1}\right) \leq\left(C_{d}\right)^{k} \mu\left(B_{1}\right) .
$$

Similarly, we have

$$
\mu\left(B_{1}\right) \leq \mu\left(2^{k} B_{2}\right) \leq\left(C_{d}\right)^{k} \mu\left(B_{2}\right) .
$$

The lemma follows.

\section{Partition of Unity}

Let $\psi$ be a smooth function on $[0, \infty)$ which satisfies $0 \leq \psi \leq 1$, and is equal to 1 on $[0,1]$ and to 0 on $[4 / 3, \infty), B_{i}$ as in Lemma 5 and $r_{i}=r\left(x_{i}\right)=\frac{1}{1000} \rho\left(x_{i}, \partial \Omega\right)$.

Taking $\varphi_{i}(x)=\psi\left(\frac{\rho\left(x, x_{i}\right)}{3 r_{i}}\right)$, we have

$$
\left.\varphi_{i}\right|_{B\left(x_{i}, 3 r_{i}\right)} \equiv 1,\left.\varphi_{i}\right|_{\left[B\left(x_{i}, 4 r_{i}\right)\right]^{c}} \equiv 0,
$$

and $\varphi_{i}$ is Lipschitz continuous, whose Lipschitz constant is $C r_{i}^{-1}$. Then, taking $\theta_{i}(x)=\frac{\varphi_{i}(x)}{\sum \varphi_{k}(x)}$, we have

$$
\operatorname{supp} \theta_{i} \subset B\left(x_{i}, 4 r_{i}\right),
$$

and $\theta_{i}$ is also Lipschitz continuous with the same Lipschitz constant $C r_{i}^{-1}$. This is proven in following.

Let $\Lambda_{i}=\left\{j \mid 4 B_{i} \cap 4 B_{j} \neq \emptyset\right\}$. For each $x \in 4 B_{i}$, there exists an $l \in \Lambda_{i}$ such that $3 B_{l} \ni x$, hence $4 B_{l} \ni x$, since $\left\{3 B_{j}\right\}$ covers $\Omega$. Apparently, $\sum_{k \in \Lambda_{i}} \varphi_{k}(x) \geq 1$, because $\varphi_{l}(x)=1$. Hence, taking any points $x, y \in 4 B_{i}$, we have

$$
\begin{aligned}
\left|\theta_{i}(x)-\theta_{i}(y)\right| & =\left|\frac{\varphi_{i}(x)}{\sum_{k \in \Lambda_{i}} \varphi_{k}(x)}-\frac{\varphi_{i}(y)}{\sum_{k \in \Lambda_{i}} \varphi_{k}(y)}\right| \\
& =\frac{\left|\varphi_{i}(x) \sum_{k \in \Lambda_{i}} \varphi_{k}(y)-\varphi_{i}(y) \sum_{k \in \Lambda_{i}} \varphi_{k}(x)\right|}{\sum_{k \in \Lambda_{i}} \varphi_{k}(x) \sum_{k \in \Lambda_{i}} \varphi_{k}(y)} \\
& \leq\left|\varphi_{i}(x) \sum_{k \in \Lambda_{i}} \varphi_{k}(y)-\varphi_{i}(y) \sum_{k \in \Lambda_{i}} \varphi_{k}(x)\right| \\
& =\left|\left[\varphi_{i}(x)-\varphi_{i}(y)\right] \sum_{k \in \Lambda_{i}} \varphi_{k}(y)+\varphi_{i}(y) \sum_{k \in \Lambda_{i}}\left[\varphi_{k}(y)-\varphi_{k}(x)\right]\right| \\
& \leq C N_{4} r_{i}^{-1} \rho(x, y)+C \sum_{k \in \Lambda_{i}} r_{k}^{-1} \rho(x, y),
\end{aligned}
$$

where $N_{4}$ is the covering mutiplicity in Lemma 5. Since $r_{k}\left(k \in \Lambda_{i}\right)$ and $r_{i}$ are comparable by Lemma 4, there exists a positive constant $A$, such that $r_{k}^{-1} \leq A r_{i}^{-1}$. Applying it to the above inequality, we reach

$$
\left|\theta_{i}(x)-\theta_{i}(y)\right| \leq C N_{4} r_{i}^{-1} \rho(x, y)+C A \sum_{k \in \Lambda_{i}} r_{i}^{-1} \rho(x, y)=\bar{C} r_{i}^{-1} \rho(x, y),
$$

where $\bar{C}=C N_{4}(1+A)$. Hence the Lipschitz constant of $\theta_{i}$ is $\bar{C} r_{i}^{-1}$.

Let $\tilde{B}=B(\tilde{x}, \tilde{r}) \subseteq \Omega$ with $200 \tilde{B} \subset \Omega$. Fixing $y \in \tilde{B}$, and applying Lemma 5 to $\Omega_{y}=\Omega \backslash\{y\}$, we get that there exists a sequence $\left\{x_{i} \in \Omega_{y} \mid i \in I \subseteq \mathbb{N}\right\}$, such that the members of the family of balls

$$
\mathcal{B}_{0}=\left\{B_{i}=B\left(x_{i}, r_{i}\right) \mid r_{i}=\frac{1}{1000} \rho\left(x_{i}, \partial \Omega_{y}\right)\right\}
$$


are mutually disjoint and such that $3 \mathcal{B}_{0}=\left\{3 B_{i}\right\}$ is an open covering of $\Omega_{y}$, the multiplicity of which is no more than $N_{3}$. Let $\left\{\theta_{i}^{y}\right\}_{i \in I}$ be the partition of unity subordinated to this covering. Then $\operatorname{supp} \theta_{i}^{y} \subset 3 B_{i}$, where $\left\{B_{i} \mid i \in I\right\}$ is the maximal disjoint family of balls of $\Omega_{y}$. Apparently, the radius of $B_{i}$ satisfies the following inequality

$$
r_{i}=\frac{1}{1000} \rho\left(x_{i}, \partial \Omega_{y}\right) \leq \frac{1}{1000} \rho\left(x_{i}, \partial \Omega\right) \equiv r\left(x_{i}\right) .
$$

Let $I^{\prime} \subset I$ be an index set of $i$ 's satisfying that $\operatorname{supp} \theta_{i}^{y} \cap 4 \tilde{B} \neq \emptyset$. Note that $i \in I^{\prime}$ implies $3 B_{i} \cap 4 \tilde{B} \neq \emptyset$. We have

Lemma 7 If $i \in I^{\prime}$, then $\rho\left(x_{i}, \partial \Omega\right)>\rho\left(x_{i}, y\right)$, and hence $r_{i}=\frac{1}{1000} \rho\left(x_{i}, y\right)$.

Proof. By $200 \tilde{B} \subset \Omega$, we get $200 \tilde{r} \leq \rho(\tilde{x}, \partial \Omega)$, and hence $r(\tilde{x})=\frac{1}{1000} \rho(\tilde{x}, \partial \Omega) \geq \frac{1}{5} \tilde{r}$. Therefore we have

$$
3 B\left(x_{i}, r\left(x_{i}\right)\right) \cap 20 B(\tilde{x}, r(\tilde{x})) \supseteq 3 B_{i} \cap 4 \tilde{B} \neq \emptyset .
$$

From (33) and Lemma 4, one can arrive at

$$
\frac{1000-20}{1000+3} r(\tilde{x}) \leq r\left(x_{i}\right) \leq \frac{1000+20}{1000-3} r(\tilde{x}) .
$$

On the other hand, by $3 B_{i} \cap 4 \tilde{B} \neq \emptyset$ and (32) we have

$$
\begin{aligned}
\rho\left(x_{i}, y\right) & \leq \rho\left(x_{i}, \tilde{x}\right)+\rho(\tilde{x}, y) \\
& <3 r_{i}+4 \tilde{r}+\tilde{r} \\
& \leq 3 r\left(x_{i}\right)+5 r(\tilde{x}) .
\end{aligned}
$$

Therefore, we have

$$
\begin{aligned}
\rho\left(x_{i}, y\right) & <3 r\left(x_{i}\right)+5 r(\tilde{x}) \leq\left(3+5 \times \frac{1003}{980}\right) r\left(x_{i}\right) \\
& =\left(6+5 \times \frac{1003}{980}\right) \cdot \frac{1}{1000} \rho\left(x_{i}, \partial \Omega\right) \\
& <\rho\left(x_{i}, \partial \Omega\right),
\end{aligned}
$$

by (34) and (35).

Lemma 8 If $i \in I^{\prime}$, then $3 B_{i} \subset 8 \tilde{B}$.

Proof. There are two points of $3 B_{i}$ located at two sides of $8 \tilde{B} \backslash 4 \tilde{B}$ separately, provided $3 B_{i} \not \subset 8 \tilde{B}$, because $3 B_{i} \cap 4 \tilde{B} \neq$ $\emptyset$. Letting the two points be $x, z$ respectively, then we have $6 r_{i} \geq \rho(z, x) \geq 4 \tilde{r}$.

Let $x \in 3 B_{i} \cap 4 \tilde{B}$, then we have $\rho(x, y) \leq \rho(x, \tilde{x})+\rho(\tilde{x}, y)<5 \tilde{r}$, and hence $6 r_{i} \geq 4 \tilde{r}>\frac{4}{5} \rho(x, y)$, i.e. $\rho(x, y)<\frac{15}{2} r_{i}$. On the other hand, by Lemma 7 , we get

$$
\rho(x, y) \geq \rho\left(y, x_{i}\right)-\rho\left(x_{i}, x\right)>1000 r_{i}-3 r_{i}=997 r_{i},
$$

which is a contradition.

Lemma 9 In doubling space, we have $\mu(B(y, \rho(x, y))) \approx \mu\left(3 B_{i}\right)$, for $i \in I^{\prime}$ and $x \in 3 B_{i} \cap 4 \tilde{B}$.

Proof. Note $\rho(x, y) \leq \rho(x, \tilde{x})+\rho(\tilde{x}, y)<5 \tilde{r}$. On the other hand, we have $\tilde{r} \leq C r_{i}$ by Lemma 4 . Hence $\rho(x, y) \leq C^{\prime} r_{i}$. Then by Lemma 7 we get

$$
\left(\rho(x, y) \geq \rho\left(y, x_{i}\right)-\rho\left(x_{i}, x\right) \geq 1000 r_{i}-3 r_{i}=997 r_{i} .\right.
$$

Therefore $r_{i} \approx \rho(x, y)$. Because $x \in \overline{B(y, \rho(x, y))}$, we have $B(y, \rho(x, y)) \cap 3 B_{i} \neq \emptyset$. So we obtain $\mu(B(y, \rho(x, y))) \approx$ $\mu\left(3 B_{i}\right)$ by Lemma 6 .

Lemma 10 Let $i \in I^{\prime}$. If $x_{i} \in B\left(y, 2^{k-1}\right) \backslash B\left(y, 2^{k-2}\right)$ for some integer $k$, then we have $3 r_{i} \approx 2^{k}, 3 B_{i} \subset B\left(y, 2^{k}\right)$, and $\mu\left(3 B_{i}\right) \approx \mu\left(B\left(y, 2^{k}\right)\right)$ by the doubling inequality.

Proof. If $i \in I^{\prime}$, then we have $r_{i}=\frac{1}{1000} \rho\left(x_{i}, y\right)$ according to Lemma 7 . Hence we have $2^{k-2} \leq \rho\left(x_{i}, y\right)=1000 r_{i} \leq$ $2^{k-1}$, which implies that $\frac{3}{1000} \cdot 2^{k-2} \leq 3 r_{i} \leq \frac{3}{1000} \cdot 2^{k-1}$ and hence $3 r_{i} \approx 2^{k}$. For any $x \in 3 B_{i}$ we get

$$
\rho(x, y) \leq \rho\left(x, x_{i}\right)+\rho\left(x_{i}, y\right) \leq 3 r_{i}+2^{k-1} \leq \frac{3}{1000} \cdot 2^{k-1}+2^{k-1}<2^{k},
$$


therefore $3 B_{i} \subset B\left(y, 2^{k}\right)$.

On the other hand, $B\left(x_{i}, 3 r_{i}\right) \subseteq B\left(y, 2^{k}\right)$ we obtain that $B\left(x_{i}, 3 r_{i}\right) \cap B\left(y, 2^{k}\right) \neq \emptyset$. Then by Lemma 6 we get $\mu\left(3 B_{i}\right) \approx \mu\left(B\left(y, 2^{k}\right)\right)$ because $3 r_{i} \approx 2^{k}$.

Lemma 11 If $2^{k-2} \geq 9 \tilde{r}$, then no $i \in I^{\prime}$ such that $x_{i} \in B\left(y, 2^{k-1}\right) \backslash B\left(y, 2^{k-2}\right)$.

Proof. If $2^{k-2} \geq 9 \tilde{r}$, then for any $z \in 8 \tilde{B}$, we have

$$
\rho(y, z) \leq \rho(y, \tilde{x})+\rho(\tilde{x}, z) \leq \tilde{r}+8 \tilde{r}=9 \tilde{r} \text {, i.e. } z \in B(y, 9 \tilde{r}),
$$

from which we get $8 \tilde{B} \subset B(y, 9 \tilde{r}) \subset B\left(y, 2^{k-2}\right)$.

By Lemma 8 , if there were $i \in I^{\prime}$, we would have $3 B_{i} \subset 8 \tilde{B}$, and hence $3 B_{i} \subset B\left(y, 2^{k-2}\right)$, which is a contradiction to $x_{i} \in B\left(y, 2^{k-1}\right) \backslash B\left(y, 2^{k-2}\right)$.

In the following, we need the estimates of Subelliptic Green functions

Lemma 12 (Sanchez-Calle, 1984) Let G be a subelliptic Green function, then we have

$$
\begin{aligned}
|G(x, y)| & \leq C \rho(x, y)^{2} \mu(B(y, \rho(x, y)))^{-1}, \\
|X G(x, y)| & \leq C \rho(x, y) \mu(B(y, \rho(x, y)))^{-1}, \\
\left|X^{2} G(x, y)\right| & \leq C \mu(B(y, \rho(x, y)))^{-1} .
\end{aligned}
$$

Using it, we can obtain

Lemma 13 Take $\eta \in C_{0}^{\infty}(\Omega)$, such that $\eta=1$ on $2 \tilde{B}, \eta \equiv 0$ outside $4 \tilde{B}$, and $|X \eta| \leq C \tilde{r}^{-1}$. Then, for $i \in I^{\prime}$, we have

$$
\left|X_{\beta}^{x}\left(\eta(x) \theta_{i}^{y}(x) X_{\alpha}^{y} G(x, y)\right)\right| \leq C \mu(B(y, \rho(x, y)))^{-1}
$$

if $y \in \tilde{B}$.

Proof. (i) $|X \eta(x)| \leq C \rho(x, y)^{-1}$ for $y \in \tilde{B}$.

Because $\eta$ is not vanish only in $4 \tilde{B}$, we consider $x \in 4 \tilde{B}$. For $y \in \tilde{B}$, we have $\rho(x, y) \leq 5 \tilde{r}$, and hence $|X \eta| \leq \tilde{C} \tilde{r}^{-1} \leq$ $C \rho(x, y)^{-1}$.

(ii) $\left|X \theta_{i}^{y}(x)\right| \leq C \rho(x, y)^{-1}$ for $i \in I^{\prime}$ and $y \in \tilde{B}$.

We only consider $x \in 3 B_{i}$ since supp $\theta_{i}^{y} \subset 3 B_{i}$. If $i \in I^{\prime}$, then $\operatorname{supp} \theta_{i}^{y} \cap 4 \tilde{B} \neq \emptyset$, and hence $3 B_{i} \cap 4 \tilde{B} \neq \emptyset$. Now we prove $r_{i}^{-1} \leq C \rho(x, y)^{-1}$. If this is true, then by (30) we have $\left|X \theta_{i}^{y}(x)\right| \leq \tilde{C} r_{i}^{-1} \leq C \rho(x, y)^{-1}$. By Lemma 8 we have $3 B_{i} \subseteq 8 \tilde{B}$, and by Lemma 7 we have $r_{i}=\frac{1}{1000} \rho\left(x_{i}, y\right)$. Therefore, we have

$$
\rho(x, y) \leq \rho\left(x, x_{i}\right)+\rho\left(x_{i}, y\right) \leq 3 r_{i}+1000 r_{i}=1003 r_{i},
$$

from which we have $r_{i}^{-1} \leq C \rho(x, y)^{-1}$.

Taking use of (i), (ii) and the estimates of Green fuctions (see 41), we get

$$
\left|X_{\beta}^{x}\left(\eta(x) \theta_{i}^{y}(x) X_{\alpha}^{y} G(x, y)\right)\right| \leq C \mu(B(y, \rho(x, y)))^{-1} .
$$

The proof of (42) is complete.

\section{Several Important Inequalities}

\subsection{Fractional Integration Theorem}

Assume that $(\Omega, \rho, \mu)$ is a metric measure space where $\mu$ is a Borel measure on $\Omega$ such that each ball has a positive measure. For a bounded open subset $O \subset \Omega, p>0, \sigma \geq 1$ and $\varepsilon>0$, define

$$
J_{\varepsilon, p}^{\sigma, O} g(x)=\sum_{2^{i} \leq 2 \sigma \operatorname{diam} O} 2^{i \varepsilon}\left(f_{B\left(x, 2^{i}\right)}|g|^{p} \mathrm{~d} \mu\right)^{1 / p},
$$

where $f_{A}=\frac{1}{\mu(A)} \int_{A}$. The following Fractional integration theorem is obtained by Hajlasz and Koskela (1995): 
Lemma 14 Suppose that $\mu$ is a doubling measure on $V=\{x \in \Omega: \rho(x, O)<2 \sigma \operatorname{diam} O\}$, and that there exist constants $b, s>0$ such that for any $x \in O$ and $r \leq 2 \sigma \operatorname{diam} O$, the following inequality holds:

$$
\mu(B(x, r)) \geq b\left(\frac{r}{\operatorname{diam} O}\right)^{s} \mu(O) .
$$

If $\varepsilon>0$ and $0<p<q<s / \varepsilon$, then we have

$$
\left\|J_{\varepsilon, p}^{\sigma, O} g\right\|_{L q^{*}(O, \mu)} \leq C(\operatorname{diam} O)^{\varepsilon} \mu(O)^{-\varepsilon / s}\|g\|_{L^{q}(V, \mu)},
$$

where, $q^{*}=s q /(s-\varepsilon q)$ and $C=C\left(\varepsilon, \sigma, p, q, b, s, C_{d}\right)$.

4.2 Subelliptic Sobolev Inequality and Poincare Inequality

The following subelliptic Sobolev inequality can be found in many papers (see for example Hajlasz \& Strzelecki, 1998):

Lemma 15 Let the homogeneous dimension of a bounded domain $\Omega \subseteq \mathbb{R}^{m}$ be $Q$, and $1 \leq p<Q$, then there exists a constant $C>0$, such that for each ball $B=B(x, r) \subseteq \Omega$, the following inequality holds:

$$
\left(f_{B}\left|u-u_{B}\right|^{p^{*}}\right)^{1 / p^{*}} \leq C r\left(f_{B}|X u|^{p}\right)^{1 / p},
$$

where $\mu$ is the Lebesgue measure, $p^{*}=Q p /(Q-p)$.

Especially, taking $p=\frac{Q^{2}}{Q+1}$ in (48) yields $p^{*}=Q^{2}$ and

$$
\left(f_{B}\left|u-u_{B}\right|^{Q^{2}}\right)^{\frac{1}{Q^{2}}} \leq C r\left(f_{B}|X u|^{\frac{Q^{2}}{Q+1}}\right)^{\frac{Q+1}{Q^{2}}} .
$$

The subelliptic Sobolev inequality implies the following Poincare inequality (see Hajlasz \& Strzelecki, 1998; Jerison, 1986):

Lemma 16 We have

$$
\int_{B}\left|u-u_{B}\right|^{p} \leq C r^{p} \int_{B}|X u|^{p} .
$$

\section{Dual Inequality of Hajlasz-Strzelecki Type}

Let $\Omega \subseteq \mathbb{R}^{m}$ be a bounded domain, $u$ : $\Omega \rightarrow S^{n}$ a weakly subelliptic $Q$-harmonic map. Denote $V_{i}=|X u|^{Q-2} X u_{i}$. Because $\sum u_{l}^{2}=1$, one can get $\sum u_{l} V_{l}=0$. Therefore, we have

$$
V_{i}=\sum u_{l}\left(u_{l} V_{i}-u_{i} V_{l}\right)
$$

Leting $E_{i, l}=u_{l} V_{i}-u_{i} V_{l} \in L^{Q /(Q-1)}$, then $X^{*} E_{i, l}=0$ (see Hajlasz \& Strzelecki, 1998), and

$$
X^{*}\left(|X u|^{Q-2} X u_{i}\right)=\sum X^{*}\left(u_{l} E_{i, l}\right) .
$$

Here, $X^{*} \xi$ is the subelliptic divergence of $\xi$, and $X u$ is the subelliptic gradient of $u$.

For a regularity of such a map $u$, the following dual inequality is established in (Hajlasz \& Strzelecki, 1998):

Lemma 17 (H-S dual inequality, Hajlasz \& Strzelecki, 1998) For any $i, l \in\{1,2, \cdots, n\}$, any ball $\tilde{B}$ with $200 \tilde{B} \subseteq \Omega$, and each function $\varphi \in M_{0}^{1, Q}(\tilde{B})$, there holds the following inequality:

$$
\left|\int_{\tilde{B}} X^{*}\left(u_{l} E_{i, l}\right)(x) \varphi(x) d x\right| \leq C\|X u\|_{L^{Q}(100 \tilde{B})}^{Q}\|X \varphi\|_{L^{Q}(\tilde{B})},
$$

where $C$ is a constant independent of $\tilde{B}$.

In order to discuss the regularity of weak solutions to more general PDEs in this paper, we need the following dual inequality of $\mathrm{H}-\mathrm{S}$ type: 
Lemma 18 (dual inequality of H-S type) Let $\tilde{B}=B(\tilde{x}, \tilde{r})$ be a ball with $200 \tilde{B} \subseteq \Omega$, and $\xi=\sum \xi_{\alpha} X_{\alpha}$ a vector field on $\Omega$ depending on $x, u, X u$, which satisfies that

$$
|\xi| \leq C|X u|^{Q-1}, X^{*} \xi=0 .
$$

If $\phi \in W_{0}^{1, Q}\left(\tilde{B}, \mathbb{R}^{K}\right)$ and $Y$ is $\mathbb{R}^{K}$-valued function defined on $\mathbb{R}^{K}$ smoothly, then there exists a constant $C$ independent of $\tilde{B}$, such that

$$
\left|\int_{\tilde{B}}\left\langle X^{*}((Y \circ u) \xi), \phi\right\rangle\right| \leq C\|X u\|_{L^{Q}(100 \tilde{B})}^{Q}\|X \phi\|_{L^{Q}(\tilde{B})},
$$

where $u \in W^{1, Q}\left(\Omega, \mathbb{R}^{K}\right)$ and $\langle\cdot, \cdot\rangle$ is the inner product of $\mathbb{R}^{K}$.

Proof. Take $\eta \in C_{0}^{\infty}(\Omega)$, such that $\eta=1$ on $2 \tilde{B}, \eta \equiv 0$ outside $4 \tilde{B}$, and $|X \eta| \leq C \tilde{r}^{-1}$. For any $\phi \in W_{0}^{1, Q}\left(\tilde{B}, \mathbb{R}^{K}\right)$, we have

$$
\phi(x)=\int_{\tilde{B}} \sum X_{\beta} G(x, \cdot) X_{\beta} \phi(\cdot),
$$

where $X_{\beta}$ stands the derivative in "." along $X_{\beta}$ and $G(x, y)$ is the Geen function. Therefore, we have

$$
\begin{aligned}
\int_{\tilde{B}}\left\langle X^{*}((Y \circ u) \xi)(x), \phi(x)\right\rangle & =\int_{\tilde{B}}\left\langle X^{*}((Y \circ u) \xi)(x), \eta(x) \phi(x)\right\rangle \\
& =\int\left\langle X^{*}((Y \circ u) \xi)(x), \eta(x) \phi(x)\right\rangle \\
& =\iint\left\langle X^{*}((Y \circ u) \xi)(x), \eta(x) \sum X_{\beta}^{y} G(x, y) X_{\beta}^{y} \phi(y)\right\rangle \mathrm{d} y \mathrm{~d} x .
\end{aligned}
$$

Here and below $X_{\beta}^{y}$ stands the derivative in " $y$ " along $X_{\beta}$. Set

$$
A_{\beta}(y)=\int X^{*}((Y \circ u) \xi)(\cdot) \eta(\cdot) X_{\beta}^{y} G(\cdot, y) .
$$

Then we have

$$
\int_{\tilde{B}}\left\langle X^{*}((Y \circ u) \xi), \phi\right\rangle=\int \sum\left\langle A_{\beta}(y), X_{\beta}^{y} \phi(y)\right\rangle \mathrm{d} y .
$$

Fix a point $y \in \tilde{B}$, and let $\left\{\theta_{i}^{y}\right\}_{i \in I}$ be a partition unity of $\Omega_{y}=\Omega \backslash\{y\}$ subordinated to the above covering. Then $\operatorname{supp} \theta_{i}^{y} \subset 3 B_{i}$, where $\left\{B_{i} \mid i \in I\right\}$ is a maximal disjoint family of balls of $\Omega_{y}$.

Let $x_{0}$ be an arbitrary point in $\Omega$ and $Y_{0}=Y\left(u_{3 B_{i}}\right)$ where $u_{3 B}=f_{3 B} u$. By the assumption $X^{*} \xi=\sum X_{\alpha}^{*} \xi_{\alpha}=0$, we have

$$
\begin{aligned}
A_{\beta}(y) & =\sum_{i \in I} \int_{3 B_{i}} X^{*}((Y \circ u) \xi)(\cdot) \eta(\cdot) \theta_{i}^{y}(\cdot) X_{\beta}^{y} G(\cdot, y) \\
& =\sum_{i \in I} \int_{3 B_{i}} X^{*}\left(\left(Y \circ u-Y_{0}\right) \xi\right)(\cdot) \eta(\cdot) \theta_{i}^{y}(\cdot) X_{\beta}^{y} G(\cdot, y) \\
& =\sum_{i \in I} \int_{3 B_{i}} \sum X_{\alpha}^{*}\left[\left(Y \circ u-Y_{0}\right) \xi_{\alpha}\right](\cdot) \eta(\cdot) \theta_{i}^{y}(\cdot) X_{\beta}^{y} G(\cdot, y) \\
& =\sum_{i \in I} \int_{3 B_{i}}\left[Y(u(\cdot))-Y\left(u_{3 B_{i}}\right)\right] \sum \xi_{\alpha}(\cdot) X_{\alpha}\left[\eta(\cdot) \theta_{i}^{y}(\cdot) X_{\beta}^{y} G(\cdot, y)\right] .
\end{aligned}
$$

Because supp $\eta \subseteq 4 \tilde{B}$, we choose $I^{\prime} \subset I$ to be a set of index $i$ 's which satisfy that $\operatorname{supp} \theta_{i}^{y} \cap 4 \tilde{B} \neq \emptyset$. Take $I_{k}^{\prime} \subseteq I^{\prime}$, the index $i$ in which satisfies that $x_{i} \in B\left(y, 2^{k-1}\right) \backslash B\left(y, 2^{k-2}\right)$. Then $I^{\prime}$ is the disjoint union of all $I_{k}^{\prime}$. Hence we have

$$
\begin{aligned}
A_{\beta}(y) & =-\sum_{k} \sum_{i \in I_{k}^{\prime}} \int_{3 B_{i}}\left[Y(u(\cdot))-Y\left(u_{3 B_{i}}\right)\right] \sum_{\alpha} \xi_{\alpha}(\cdot) X_{\alpha}\left[\eta(\cdot) \theta_{i}^{y}(\cdot) X_{\beta}^{y} G(\cdot, y)\right] \\
& =-\sum_{2^{k-2} \leq 9 \tilde{r}} \sum_{i \in I_{k}^{\prime}} \int_{3 B_{i}}\left[Y(u(\cdot))-Y\left(u_{3 B_{i}}\right)\right] \sum_{\alpha} \xi_{\alpha}(\cdot) X_{\alpha}\left[\eta(\cdot) \theta_{i}^{y}(\cdot) X_{\beta}^{y} G(\cdot, y)\right],
\end{aligned}
$$


where the second equality holds because of Lemma 11.

Applying (42) to (58), and taking use of Lemma 9, we have

$$
\begin{aligned}
\left|A_{\beta}(y)\right| & \leq C \sum_{2^{k-2} \leq 9 \tilde{r}} \sum_{i \in I_{k}^{\prime}} \int_{3 B_{i}} \frac{1}{\mu(B(y, \rho(\cdot, y)))}\left|Y(u(\cdot))-Y\left(u_{3 B_{i}}\right)\right| \xi \mid \\
& \leq C \sum_{2^{k-2} \leq 9 \tilde{r}} \sum_{i \in I_{k}^{\prime}} f_{3 B_{i}}\left|Y(u(\cdot))-Y\left(u_{3 B_{i}}\right)\right| \xi \mid \\
& \leq C \sum_{2^{k-2} \leq 9 \tilde{r}} \sum_{i \in I_{k}^{\prime}}\left(f_{3 B_{i}}\left|Y(u(\cdot))-Y\left(u_{3 B_{i}}\right)\right|^{Q^{2}}\right)^{\frac{1}{Q^{2}}}\left(f_{3 B_{i}}|\xi|^{\frac{Q^{2}}{Q^{2}-1}}\right)^{\frac{Q^{Q^{2}-1}}{Q^{2}}} \\
& \leq C \sup |D Y| \sum_{2^{k-2} \leq 9 \tilde{r}} \sum_{i \in I_{k}^{\prime}}\left(f_{3 B_{i}}\left|u(\cdot)-u_{3 B_{i}}\right|^{Q^{2}}\right)^{\frac{1}{Q^{2}}}\left(f_{3 B_{i}}|\xi|^{\frac{Q^{2}}{Q^{2}-1}}\right)^{\frac{Q^{2}-1}{Q^{2}}} \\
& \leq C \sup |D Y| \sum_{2^{k-2} \leq 9 \tilde{r}} \sum_{i \in I_{k}^{\prime}} r_{i}\left(f_{3 B_{i}}|X u|^{\frac{Q^{2}}{Q^{2+1}}}\right)^{\frac{Q+1}{Q^{2}}}\left(f_{3 B_{i}}|X u|^{\frac{Q^{2}}{Q+1}}\right)^{\frac{Q^{2}-1}{Q^{2}}} \\
& \leq C \sup |D Y| \sum_{2^{k-2} \leq 9 \tilde{r}} \sum_{i \in I_{k}^{\prime}} r_{i}\left(f_{3 B_{i}}|X u|^{\frac{Q^{2}}{Q^{2}+1}}\right)^{\frac{Q+1}{Q}}
\end{aligned}
$$

where we have used Sobolev Inequality (49). If $x_{i} \in B\left(y, 2^{k-1}\right) \backslash B\left(y, 2^{k-2}\right)$ for some integer $k$, then from Lemma 10 we get

$$
r_{i}\left(f_{3 B_{i}}|X u|^{\frac{Q^{2}}{Q+1}}\right)^{\frac{Q+1}{Q}} \leq C 2^{k}\left(f_{B\left(y, 2^{k}\right)}|X u|^{\frac{Q^{2}}{Q+1}}\right)^{\frac{Q+1}{Q}} .
$$

Furthermore, let $N$ be the number of index $i \in I^{\prime}$ such that $x_{i} \in B\left(y, 2^{k-1}\right) \backslash B\left(y, 2^{k-2}\right)$ has a upper bound depending only on $p$ and $\Omega$.

Substituting (60) into (59) yields

$$
\left|A_{\alpha}(y)\right| \leq C \sum_{k} \sum_{i \in I_{k}^{\prime}} 2^{k}\left(f_{B\left(y, 2^{k}\right)}|X u|^{\frac{Q^{2}}{Q^{2}+1}}\right)^{\frac{Q+1}{Q}} \leq C \sum_{2^{k} \leq 2 \cdot 2 \cdot 16 \tilde{r}} 2^{k}\left(f_{B\left(y, 2^{k}\right)}|X u|^{\frac{Q^{2}}{Q+1}}\right)^{\frac{Q+1}{Q}}=C J_{1, \frac{Q}{Q+1}, 8 \tilde{B}}\left(|X u|^{Q}\right)(y) .
$$

Take

$$
\varepsilon=1, \sigma=2, q^{*}=\frac{Q}{Q-1}, q=1, p=\frac{Q}{Q+1}, s=Q, O=8 \tilde{B},
$$

From fractional integration formula (47) we have (for $O=8 \tilde{B}$, the conditions of Lemma 14 are satisfied):

$$
\left\|J_{1, \frac{Q}{Q+1}}^{2, B_{8 r}\left(x_{0}\right)}|X u|^{Q}\right\|_{L^{Q /(Q-1)}(8 \tilde{B})} \leq C\|X u\|_{L^{1}(V)}^{Q},
$$

where $V=\{x: \rho(x, 8 \tilde{B})<2 \cdot 2 \cdot 16 \tilde{r}\}=72 \tilde{B}$. Hence we have

$$
\left\|A_{\alpha}\right\|_{L^{Q / Q-1)}(8 \tilde{B})} \leq C\|X u\|_{L^{Q}(72 \tilde{B})}^{Q},
$$

from which wed get

$$
\left\|A_{\alpha}\right\|_{L^{Q /(Q-1)}(\tilde{B})} \leq\left\|A_{\alpha}\right\|_{L^{Q / Q-1)}(8 \tilde{B})} \leq C\|X u\|_{L^{Q(72 \tilde{B})}}^{Q} \leq C\|X u\|_{L^{Q(100 \tilde{B})}}^{Q} .
$$

Applying (65) to (56), we get

$$
\begin{aligned}
\int_{\tilde{B}}\left\langle X^{*}((Y \circ u) \xi), \phi\right\rangle & =\int \sum\left\langle A_{\beta}(y), X_{\beta}^{y} \phi(y)\right\rangle \mathrm{d} y \\
& \leq \sum\left\|A_{\beta}\right\|_{L^{Q /(Q-1)}(\tilde{B})}\|X \phi\|_{L^{Q}(\tilde{B})} \\
& \leq C\|X u\|_{L^{Q}(100 \tilde{B})}^{Q}\|X \phi\|_{L^{Q(\tilde{B})}}
\end{aligned}
$$


which is we need.

\section{Regularity of Weak Solutions to a Subelliptic PDE System of Divergence Type-Proof of Lemma 1}

For each $x_{0} \in \Omega$, we take a small ball $B_{r}\left(x_{0}\right)$. Let $\eta$ be a cut-off function which is 1 on $B_{r}\left(x_{0}\right)$, and is zero outside $B_{2 r}\left(x_{0}\right)$, and furthmore $|X \eta| \leq C r^{-1}$. Let $\psi=\eta\left(u-u_{2 r}\right)$, where $u_{2 r}=\frac{1}{\mu\left(B_{2 r}\left(x_{0}\right)\right)} \int_{B_{2 r}\left(x_{0}\right)} u \mathrm{~d} x \equiv f_{B_{2 r}\left(x_{0}\right)} u \mathrm{~d} x$. Testing (5) by $\psi$ yields

$$
\int_{B_{2 r}\left(x_{0}\right)}\left\langle\sum X_{\alpha}^{*}\left(A_{\alpha \beta} X_{\beta} u\right), \psi\right\rangle=\int_{B_{2 r}\left(x_{0}\right)}\left\langle\sum X_{\alpha}^{*}\left(\xi_{\alpha, i} Y_{i} \circ u\right), \psi\right\rangle .
$$

Applying (Lemma 18), the dual inequality of H-S type to the right hand side of (67) yields

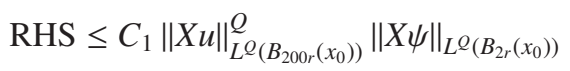

$$
\begin{aligned}
& \leq C_{2}\|X u\|_{L^{Q}\left(B_{200 r}\left(x_{0}\right)\right)}^{Q}\|X u\|_{L^{Q}\left(B_{2 r}\left(x_{0}\right)\right)} .
\end{aligned}
$$

Then, applying the Poincare inequality (50) to estimate the left hand side of (67), we have

$$
\begin{aligned}
\text { LHS } & =\int_{B_{2 r}\left(x_{0}\right)} \sum A_{\alpha \beta}\left\langle X_{\beta} u, X_{\alpha} \psi\right\rangle \\
& =\int_{B_{2 r}\left(x_{0}\right)} \sum A_{\alpha \beta}\left\langle X_{\beta} u, X_{\alpha} u\right\rangle \eta+\int_{B_{2 r}\left(x_{0}\right)} \sum A_{\alpha \beta}\left\langle X_{\beta} u, u-u_{2 r}\right\rangle X_{\alpha} \eta \\
& \geq C_{3} \int_{B_{r}\left(x_{0}\right)}|X u|^{Q}-\int_{B_{2 r}\left(x_{0}\right)} \sum A_{\alpha \beta}\left|X_{\alpha} \eta\right|\left|X_{\beta} u\right|\left|u-u_{2 r}\right| \\
& \geq C_{3} \int_{B_{r}\left(x_{0}\right)}|X u|^{Q}-C_{4} \int_{T_{2 r}} \sum|X u|^{Q-2}\left|X_{\alpha} \eta\right|\left|X_{\alpha} u\right|\left|u-u_{2 r}\right| \\
& \geq C_{3} \int_{B_{r}\left(x_{0}\right)}|X u|^{Q}-C_{5} r^{-1} \int_{T_{2 r}}|X u|^{Q-1}\left|u-u_{2 r}\right| \\
& \geq C_{3} \int_{B_{r}\left(x_{0}\right)}|X u|^{Q}-C_{5} r^{-1}\left(\int_{T_{2 r}}|X u|^{Q}\right)^{\frac{Q-1}{Q}}\left(\int_{B_{2 r}\left(x_{0}\right)}\left|u-u_{2 r}\right|^{Q}\right)^{\frac{1}{Q}} \\
& \geq C_{3} \int_{B_{r}\left(x_{0}\right)}|X u|^{Q}-C_{6}\left(\int_{T_{2 r}}|X u|^{Q}\right)^{\frac{Q-1}{Q}}\left(\int_{B_{2 r}\left(x_{0}\right)}|X u|^{Q}\right)^{\frac{1}{Q}},
\end{aligned}
$$

where $T_{2 r}=B_{2 r}\left(x_{0}\right)-B_{r}\left(x_{0}\right)$. Therefore we get

$$
\begin{aligned}
\int_{B_{r}\left(x_{0}\right)}|X u|^{Q} & \leq C\left(\int_{T_{2 r}}|X u|^{Q}\right)^{\frac{Q-1}{Q}}\left(\int_{B_{2 r}\left(x_{0}\right)}|X u|^{Q}\right)^{\frac{1}{Q}}+C\left(\int_{B_{200 r}\left(x_{0}\right)}|X u|^{Q}\right)\left(\int_{B_{2 r}\left(x_{0}\right)}|X u|^{Q}\right)^{\frac{1}{Q}} \\
& \leq C\left(\int_{B_{2 r}\left(x_{0}\right)}|X u|^{Q}-\int_{B_{r}\left(x_{0}\right)}|X u|^{Q}\right)^{\frac{Q-1}{Q}}\left(\int_{B_{2 r}\left(x_{0}\right)}|X u|^{Q}\right)^{\frac{1}{Q}}+C\left(\int_{B_{200 r}\left(x_{0}\right)}|X u|^{Q}\right)\left(\int_{B_{2 r}\left(x_{0}\right)}|X u|^{Q}\right)^{\frac{1}{Q}} \\
& \leq C\left(\int_{B_{200 r}\left(x_{0}\right)}|X u|^{Q}-\int_{B_{r}\left(x_{0}\right)}|X u|^{Q}\right)^{\frac{Q-1}{Q}}\left(\int_{B_{200 r}\left(x_{0}\right)}|X u|^{Q}\right)^{\frac{1}{Q}}+C\left(\int_{B_{200 r}\left(x_{0}\right)}|X u|^{Q}\right)^{\frac{Q+1}{Q}} .
\end{aligned}
$$

Let $M\left(x_{0}, r\right)=\int_{B_{r}\left(x_{0}\right)}|X u|^{Q}$. Then, there exist positive numbers $r_{0}$ and $\lambda \in(0,1)$, which are independent of $x_{0}$, such that for all $r \leq r_{0}$ the following inequality holds

$$
M\left(x_{0}, r\right) \leq \lambda M\left(x_{0}, 200 r\right) .
$$

In fact, if the inequality were not true, there would be a positive $r \leq r_{0}$, such that $M\left(x_{0}, r\right)>\lambda M\left(x_{0}, 200 r\right)$ for all $r_{0}>0$ and $\lambda \in(0,1)$. Hence we have

$$
\lambda M\left(x_{0}, 200 r\right)<C(1-\lambda)^{\frac{Q-1}{Q}} M\left(x_{0}, 200 r\right)+C M\left(x_{0}, 200 r\right)^{\frac{Q+1}{Q}} .
$$

For $\lambda \in[1 / 2,1)$ and positive number $r_{0}$ small enough, we have

$$
\frac{1}{2}<C(1-\lambda)^{(Q-1) / Q}+C M\left(x_{0}, 200 r\right)^{1 / Q} .
$$


for arbitrararily small positive number $r$. Letting $\lambda$ tend to 1 , and $r$ tend to 0 yield a contradiction.

Then, by a standard calculation from (71) we obtain $\int_{B\left(x_{1}, r\right)}|X u|^{Q} \leq C r^{\mu}$ for any $x_{1} \in B_{r_{0}}\left(x_{0}\right)$ and any $r \leq r_{0}$, which implies that $u$ is locally Holder continuous (see Hajlasz \& Strzelecki, 1998).

\section{Regularity of Weakly Subelliptic F-Harmonic Maps-Proof of Theorem 2}

In this section, we deduce the regularity of weakly subelliptic $F$-harmonic maps.

Let $v_{n+1}, \cdots, v_{K}$ be a local field of normal frame of $N$ in $\mathbb{R}^{K}$, and $A^{k}(X, Y)=X\left(v_{k}\right) \cdot Y$ is the second fundamental form of $N$ in $\mathbb{R}^{K}$ respect to $v_{k}$. Let $\Delta_{X}^{F} u=\sum X_{\alpha}^{*}\left(F^{\prime}\left(\frac{|X u|^{2}}{2}\right) X_{\alpha} u\right)$ be the $F$-Laplacian of $u$. Then the Euler-Lagrange equation of weakly subelliptic $F$-harmonic maps can be written in the following form:

$$
\Delta_{X}^{F} u=F^{\prime}\left(\frac{|X u|^{2}}{2}\right) A(u)(X u, X u),
$$

i.e.

$$
\sum X_{\alpha}^{*}\left(F^{\prime}\left(\frac{|X u|^{2}}{2}\right) X_{\alpha} u\right)=F^{\prime}\left(\frac{|X u|^{2}}{2}\right) A(u)(X u, X u)
$$

where

$$
A(u)(X u, X u)=\sum_{k=n+1}^{v} \sum_{\alpha} A^{k}(u)\left(X_{\alpha} u, X_{\alpha} u\right)\left(v_{k} \circ u\right) .
$$

For example, if $N=S^{n} \subset \mathbb{R}^{n+1}$, then the Euler-Lagrange equation is

$$
\Delta_{X}^{F} u=-F^{\prime}\left(\frac{|X u|^{2}}{2}\right)|X u|^{2} u
$$

On $N$, we call a vector field $K$ is of Killing, if $\left\langle Z, \nabla_{Z} K\right\rangle=0$ for any vector field $Z$, or equivalently $\left\langle Y, \nabla_{Z} K\right\rangle+$ $\left\langle Z, \nabla_{Y} K\right\rangle=0$ for any vector fields $Y, Z$, where $\langle\cdot, \cdot\rangle$ is the Riemannian inner product of $N$.

Lemma 19 Let $u \in W^{1, Q}(\Omega, N)$ be a weakly subelliptic F-harmonic map, $K$ a Killing vector field of $N$, and

$$
\xi=F^{\prime}\left(|X u|^{2} / 2\right) \sum\left\langle K \circ u, X_{\alpha} u\right\rangle X_{\alpha} u .
$$

Then $X^{*} \xi=0$, i.e. $\sum X_{\alpha}^{*}\left(F^{\prime}\left(|X u|^{2} / 2\right)\left\langle K \circ u, X_{\alpha} u\right\rangle\right)=0$.

Proof. For $\phi \in C_{0}^{\infty}(\Omega, \mathbb{R})$, set $\psi=\phi K \circ u \in W_{0}^{1, Q}\left(\Omega, \mathbb{R}^{K}\right)$. Applying $\psi$ to the Euler-Lagrange Equation (73), we have

$$
\begin{aligned}
0 & =\int_{\Omega}\left\langle\Delta_{X}^{F} u, \psi\right\rangle=\int_{\Omega}\left\langle\sum X_{\alpha}^{*}\left(F^{\prime}\left(|X u|^{2} / 2\right) X_{\alpha} u\right), \phi K \circ u\right\rangle \\
& =\int_{\Omega} \sum\left\langle F^{\prime}\left(|X u|^{2} / 2\right) X_{\alpha} u, X_{\alpha}(\phi K \circ u)\right\rangle \\
& =\int_{\Omega} \sum\left\langle F^{\prime}\left(|X u|^{2} / 2\right) X_{\alpha} u,\left(X_{\alpha} \phi\right) K \circ u\right\rangle+\int_{\Omega} \sum\left\langle F^{\prime}\left(|X u|^{2} / 2\right) X_{\alpha} u, \phi X_{\alpha}(K \circ u)\right\rangle .
\end{aligned}
$$

Since $K$ is a Killing field, we get $\sum\left\langle X_{\alpha} u, X_{\alpha}(K \circ u)\right\rangle=\sum\left\langle X_{\alpha} u, \nabla_{X_{\alpha} u} K\right\rangle=0$, and hence the last integral vanish. Therefore, we have

$$
0=\int_{\Omega} \sum\left\langle F^{\prime}\left(|X u|^{2} / 2\right) X_{\alpha} u, K \circ u\right\rangle X_{\alpha} \phi=\int_{\Omega}\left(X^{*} \xi\right) \phi .
$$

The follwing lemma is proven by Helein (1991a).

Lemma 20 Let $N$ be a compact Riemannian manifold where the isometric transformation group acts transitively. Then, there exist vector fields $Y_{1}, \cdots, Y_{q}$ and Killing fields $K_{1}, \cdots, K_{q}$ on $N$, such that for any vector filed $V$, we have

$$
V=\left\langle K_{1}, V\right\rangle Y_{1}+\cdots+\left\langle K_{q}, V\right\rangle Y_{q}
$$

From now on, we assume that $F^{\prime}(t) \sim t^{\frac{Q-1}{2}}$. The range of index $i$ is from 1 to $q$. 
Take $V_{\alpha}=X_{\alpha} u$ in Lemma 20. Then we have

$$
X_{\alpha} u=\sum\left\langle K_{i} \circ u, X_{\alpha} u\right\rangle Y_{i} \circ u .
$$

Let $K_{\alpha, i}=\left\langle K_{i} \circ u, X_{\alpha} u\right\rangle$. Then we get

$$
F^{\prime}\left(|X u|^{2} / 2\right) X_{\alpha} u=\sum F^{\prime}\left(|X u|^{2} / 2\right) K_{\alpha, i} Y_{i} \circ u .
$$

Because $u$ is weakly subelliptic $F$-harmonic, by Lemma 19 , we have

$$
\sum X_{\alpha}^{*}\left(F^{\prime}\left(|X u|^{2} / 2\right) K_{\alpha, i}\right)=0 .
$$

I.e.

$$
\sum X_{\alpha}^{*} \xi_{\alpha, i}=0
$$

where $\xi_{\alpha, i}=F^{\prime}\left(|X u|^{2} / 2\right) K_{\alpha, i}$.

Letting $\xi_{i}=\sum \xi_{\alpha, i} X_{\alpha}=\sum F^{\prime}\left(|X u|^{2} / 2\right)\left\langle K_{i} \circ u, X_{\alpha} u\right\rangle X_{\alpha}$, then we have $X^{*} \xi_{i}=0$. Apparently, $\left|\xi_{\alpha, i}\right| \leq C|X u|^{Q-1}$. From (78) one has

$$
\sum X_{\alpha}^{*}\left(F^{\prime}\left(|X u|^{2} / 2\right) X_{\alpha} u\right)=\sum X_{\alpha}^{*}\left(\xi_{\alpha, i} Y_{i} \circ u\right) .
$$

From this and Lemma 1, we prove Theorem 2.

\section{References}

Barletta, E., \& Dragomir, S. (2004). On the regularity of weak subelliptic F-harmonic maps. Tsukuba J. Math., $28(2), 417-436$.

Bellaiche, A., \& Risler, J.-J. (Eds.). (1996). Sub-Riemannian Geometry. Progress in Math., 144. Birkhauser. http://dx.doi.org/10.1007/978-3-0348-9210-0

Hajlasz, P., \& Koskela, P. (1995). Sobolev meets Poincare. C. R. Acad. Sci. Paris, 320, 1211-1215.

Hajlasz, P., \& Strzelecki, P. (1998). Subelliptic p-harmonic maps into spheres and the ghost of Hardy spaces. Math. Ann., 312, 341-362. http://dx.doi.org/10.1007/s002080050225

Helein, F. (1990). Regularite des applications faiblement harmonique sentre une surface et une sphere. C. R. Acad. Sci. Paris, 311, 519-524.

Helein, F. (1991a). Regularity of weakly harmonic maps from a surface into a manifold with symmetries. Manuscripta Math., 70, 203-218. http://dx.doi.org/10.1007/BF02568371

Helein, F. (1991b). Regularite des applications faiblement harmonique sentre une surface et une variete riemannienne. C. R. Acad. Sci. Paris, 312, 591-596.

Jerison, D. (1986). The Poincare inequality for vector fields satisfying Hormander's conditions. Duke Math. J., 53, 503-523. http://dx.doi.org/10.1215/S0012-7094-86-05329-9

Jost, J., \& Xu, C. J. (1998). Subelliptic harmonic maps. Trans. AMS., 350(11), 4633-4649. http://dx.doi.org/10.1090/S0002-9947-98-01992-8

Jost, J., \& Yang, Y. H. (2005). Heat flow for horizontal harmonic maps into a class of Carnot-Caratheodory spaces. Math. Res. Letters, 12, 513-529.

Sanchez-Calle, A. (1984). Fundamental solutions and geometry of the sum of squares of vector fields. Invent. Math., 78, 142-160. http://dx.doi.org/10.1007/BF01388721

Stricharts, R. S. (1986). Sub-Riemannian geometry. J. Diff. Geom., 24, 221-263.

Strzelecki, P. (1994). Regularity of p-harmonic maps from p-dimensional ball into a sphere. Manuscripta Math., 407-415. http://dx.doi.org/10.1007/BF02567710

Toro, T., \& Wang, C. Y. (1995). Compactness properties of weakly p-harmonic maps into homogeneous spaces. Indiana Math. J., 44, 87-113.

Wang, C. Y. (2003). Subelliptic harmonic maps from Carnot groups. Calc. Var., 18, 95-115. http://dx.doi.org/10.1007/s00526-002-0184-7 
Zhou, Z. R. (1999). Uniqueness of subelliptic harmonic maps. Ann. of Global Anal. and Geom., 6, 581-594. http://dx.doi.org/10.1023/A:1006620325479

Zhou, Z. R. (2005). A Note on Boundary Regularity of Subelliptic Harmonic Maps. Kodai Math. J., 28(3), 525-533. http://dx.doi.org/10.2996/kmj/1134397766

Zhou, Z. R. (2013). Heat flows of subelliptic harmonic maps into Riemannian manifolds with nonpositive curvatures. J. Geom. Anal., 23, 471-489.

\section{Copyrights}

Copyright for this article is retained by the author(s), with first publication rights granted to the journal.

This is an open-access article distributed under the terms and conditions of the Creative Commons Attribution license (http://creativecommons.org/licenses/by/3.0/). 